\title{
Rotator cuff injury: still a clinical controversy?
}

\author{
P. Hardy $\cdot$ S. Sanghavi
}

Published online: 20 February 2009

(C) Springer-Verlag 2009

The rotator cuff mechanism is the primary stabiliser of the gleno-humeral joint and is vulnerable to considerable morbidity because of its anatomy and function. Both intrinsic and extrinsic factors are responsible for rotator cuff pathology, which is a disease spectrum ranging from inflammation to full thickness tears. The most notable extrinsic factors are impingement and demographic variables, whereas age-related degeneration is the most important intrinsic factor. Failure of the rotator cuff may progress to involve the full thickness of the tendinous insertions of the supraspinatus and then may extend to involve the infraspinatus and subscapularis tendons. Tear size tends to increase with time because there is little potential for spontaneous healing. Thorough knowledge of the rotator cuff anatomy and the management of tendon failure at other sites are helpful in the approach to the management of rotator cuff tears. There is a remarkable inconsistency in perceptions among the orthopaedic surgeons about the indications for rotator cuff surgery. There is also no clear consensus in terms of the optimal management and the timing of surgical intervention of chronic degenerative rotator cuff lesions. The treating surgeon's goal should be to identify the treatment most likely to relieve pain and restore the patient to the greatest durable function possible given the extent of the individual's pathology. Decisions regarding the need for imaging should be based on whether the results are likely to affect treatment. Plain radiographs of the shoulder are helpful to assess the gleno-humeral articulation, the acromio-humeral distance and the acromion morphology. Ultrasonography

P. Hardy $(\bowtie) \cdot$ S. Sanghavi

Hopital Ambroise Pare, West Paris University,

9, Avenue Charles de Gaulle, 92100 Boulogne, France

e-mail: philippe.hardy@apr.aphp.fr
(US), MRI and MRI-arthrography are all useful to evaluate the integrity of the rotator cuff tendons. Both MRI and US are highly accurate and sensitive (90\% accuracy, 97\% sensitivities), but have modest specificity $(\sim 67 \%)$ in diagnosing full-thickness and partial-thickness tears, when compared with the findings at arthroscopy [7]. However, MRI provides high-resolution, objective anatomic details of cuff tears and is also useful to evaluate the extent of delamination, muscle atrophy and extent of fatty infiltration. T2-weighted MRI images are moreover helpful to accurately estimate the cuff tear size. With better evaluation of the tear configuration, a more accurate impression of the reparability can be obtained. Severely retracted rotator cuff should be carefully interpreted as they may only represent the apex of a "U-shaped" tear, which is very amenable to a repair. High-resolution US in experienced hands can also give results equivalent to that of MRI, but its efficacy is operator- and interpreter-dependent, and inaccuracies in the evaluation have limited its use.

The goal of rotator cuff surgery is to optimise the bond between the bone and soft tissue at the rotator cuff footprint. The cuff repair must collectively withstand physiological loads in the postoperative period while biological healing takes place. Factors that favour durable surgical repair of the rotator cuff include an age of $<60$ years, a traumatic onset of weakness, a short duration of symptoms $(<2$ months), no history of smoking, receipt of few cortisone injections, no previous shoulder surgery, stable shoulder with good range of motion and no muscle atrophy on physical examination, and MRI or US findings that show minimal retraction, good tendon quality and minimal muscle atrophy.

A recent Cochrane review showed functional outcomes after arthroscopic repair to be similar to open and miniopen repairs, however, with potential for earlier recovery 
after arthroscopic intervention [2]. Biomechanically, the rotator cuff repair can be secured by inserting a doubleloaded suture anchor in the anterior and middle parts of the greater tuberosity, which doubles the number of fixation points, consequently reducing the tension at each fixation point by $\sim 50 \%$ and improving the pullout anchor strengths. Osteopenic patients benefit from screw-type metal anchors and a greater number of anchors than would otherwise be used. Acute traumatic ruptures of the rotator cuff should be repaired as soon as possible, ideally within 6 weeks after injury [6]. Patients who present late can also benefit from cuff repair as the process of muscle atrophy and fatty infiltration is both irreversible and functionally damaging. Few studies have shown a successful cuff repair to moderately improve the degree of muscle atrophy and arrest further fatty infiltration. In contrast, failed repairs and conservative management will lead to progression of both fatty infiltration and atrophy over time [3, 8]. Acute or chronic partial-thickness cuff tears often improve with conservative management and a stretching programme. Patients with symptoms refractory to a stretching programme may benefit from cuff debridement, smoothing of the humero-scapular motion interface and cuff deep-surface curettage. Patients with degenerative full thickness chronic tears, and large and massive rotator cuff tears in patients who are minimally symptomatic with low functional demands are best managed without surgery. Subacromial corticosteroid injections should be used only in cases in which the response to other non-surgical interventions is inadequate and when surgical intervention is not an option. There is no evidence of any significant benefit of corticosteroid injection in patients with rotator cuff tears, and hence it should be discouraged. There is very little literature, and the indications and benefits are unclear on electrotherapy, therapeutic US, acupuncture, injection of hyaluronic acid and shock-wave therapy.

Degenerative rotator cuff tear is frequently associated with degeneration of the long head of the biceps, which is an important source of shoulder pain and disability and should be specifically addressed to prevent the negative functional consequences. Tenotomy or tenodesis is recommended for irreversible structural changes in the tendon, preferably tenodesis for the younger patient [1]. Tendon transfer should be considered in younger patients when rotator cuff tears are un-repairable and there is unacceptable dysfunction. Sub-coracoid pectoralis transfer for anterio-superior defects and latissimus dorsi transfers for postero-superior tears have been performed with fair to good success. Reverse shoulder arthroplasty has been helpful for those with a pseudo-paralytic shoulder even in the absence of significant gleno-humeral arthritis.

Reports on the results of surgery for rotator cuff repair indicate that more than $75 \%$ of repairs of defects that involve only the supraspinatus remain intact at 5 years and are associated with improved comfort and function, whereas $<50 \%$ of repairs of defects that involve the supraspinatus and infraspinatus remain intact at 5 years. Improved comfort may be achieved even if the repair does not remain intact [5, 6]. Interestingly, subjective and functional improvement is noted even in patients in whom the tendon repair has failed. This may be due either to subacromial decompression leading to improvements in pain and function, independent of the status of the damaged tendon, or a stricter, more intensive physical therapy regimen. With regard to cancellous bed preparation to aid tendon-bone healing, there is no evidence of improved outcomes, and further studies are required if any recommendations are to be made. A controversial question in arthroscopic rotator cuff surgery is the relative merits of single-row versus double-row repair. In vitro anatomical studies strongly suggest that a double-row repair produces a significantly larger supraspinatus footprint and better biomechanical construct than single-row repairs. However, only few clinical studies have shown any differences in the clinical scores in the single- or double-row repair groups, with 2-year follow-up, except improved radiological results in double-row repairs. Therefore, while double-row repairs give superior anatomical and biomechanical results in vivo, no current data support better subjective outcomes than single-row repairs. It is more likely that the existing studies are underpowered, and improvements in functional scores may emerge with larger studies. Recently, a new trans-osseous equivalent or suture bridge technique was reported, and it was shown that its failure load was significantly higher. This trans-osseous equivalent technique uses suture bridges between anchors to add mechanical support to the repair construct and shows considerable promise. Controversy also exists in terms of the advisability of repairing those tears that are accompanied by stage III or IV fatty infiltration. Higher repair failure rates have been documented when fatty infiltration is equal to or greater than muscle on computed tomography evaluation (Goutallier stages III and IV). Most studies have been limited by the lack of a control group, the types of outcome data reported, the lack of long-term follow-up of all prospectively enrolled patients and the questionable generalisation of studies performed by expert surgeons in major centres to general practice. Randomised trials are needed to assess and compare conservative with surgical interventions and to assess different surgical procedures.

Over the next decade, biologic enhancements to healing will play a major role in increasing the success rate of rotator cuff healing. Biological alternatives, such as autologus cell regeneration, acellular biological scaffolds, gene transfection via adenovirus vectors, cuff replacement with biological tissues, augmentation of the repaired rotator cuff 
with fibroblast or stem cell therapy, and augmentation with materials like porcine small intestinal submucosa (PSIS) grafts, human/animal skin, muscle auto/allograft as well as several synthetic materials have been proposed. Biologic augmentation of massive tears as compared to non-massive tears may be particularly valuable as they often have poorer long-term outcomes. However, to date there is only a single study on this subject, and further studies are required [4]. All the above techniques are still being developed and need further investigation with long follow-up. These techniques have to be first explored in animal studies before any clinical exposure can be considered. We also need to develop the technical expertise before these biological therapies gain widespread acceptability.

\section{References}

1. Boileau P, Baqué F, Valerio L et al (2007) Isolated arthroscopic biceps tenotomy or tenodesis improves symptoms in patients with massive irreparable rotator cuff tears. J Bone Joint Surg Am 89:747-757
2. Coghlan J, Buchbinder R, Green S et al (2008) Surgery for rotator cuff disease. Cochrane Database Syst Rev 1:CD005619

3. Gerber C, Schneeberger AG, Hoppeler H, Meyer DC (2007) Correlation of atrophy and fatty infiltration on strength and integrity of rotator cuff repairs: a study in thirteen patients. J Shoulder Elbow Surg 16:691-696

4. Iannotti JP, Codsi MJ, Kwon YW et al (2006) Porcine small intestine sub mucosa augmentation of surgical repair of chronic two-tendon rotator cuff tears. A randomized, controlled trial. J Bone Joint Surg Am 88:1238-1244

5. Lafosse L, Brozska R, Toussaint B, Gobezie R (2007) The outcome and structural integrity of arthroscopic rotator cuff repair with use of the double-row suture anchor technique. J Bone Joint Surg Am 89:1533-1541

6. Sugaya H, Maeda K, Matsuki K, Moriishi J (2007) Repair integrity and functional outcome after arthroscopic double-row rotator cuff repair: a prospective outcome study. J Bone Joint Surg Am 89:953-960

7. Teefey SA, Rubin DA, Middleton WD et al (2004) Detection and quantification of rotator cuff tears: comparison of ultrasonographic, magnetic resonance imaging, and arthroscopic findings in seventy-one consecutive cases. J Bone Joint Surg Am 86:708-711

8. Zingg PO, Jost B, Sukthankar A, Buhler M et al (2007) Clinical and structural outcomes of nonoperative management of massive rotator cuff tears. J Bone Joint Surg Am 89:1928-1934 